\title{
Drosophila CLASP is required for the incorporation of microtubule subunits into fluxing kinetochore fibres
}

\author{
Helder Maiato ${ }^{1}$, Alexey Khodjakov ${ }^{1,2,3}$, and Conly L. Rieder ${ }^{1,2,3,4}$ \\ 1 Laboratory of Cell Regulation, Wadsworth Center, New York State Department of Health, Empire State \\ Plaza, Albany, NY 12201-0509, USA \\ 2Department of Biomedical Sciences, State University of New York, Albany, NY 12222, USA \\ 3Marine Biological Laboratory, Woods Hole, MA 02543, USA
}

\section{Abstract}

The motion of a chromosome during mitosis is mediated by a bundle of microtubules, termed a kinetochore fibre (K-fibre), which connects the kinetochore of the chromosome to a spindle pole. Once formed, mature K-fibres maintain a steady state length because the continuous addition of microtubule subunits onto microtubule plus ends at the kinetochore is balanced by their removal at their minus ends within the pole. This condition is known as 'microtubule poleward flux' 1 .

Chromosome motion and changes in position are then driven by changes in K-fibre length, which in turn are controlled by changes in the rates at which microtubule subunits are added at the kinetochore and/or removed from the pole ${ }^{2}$. A key to understanding the role of flux in mitosis is to identify the molecular factors that drive it. Here we use Drosophila melanogaster S2 cells expressing $\alpha$-tubulin tagged with green fluorescent protein, RNA interference, laser microsurgery and photobleaching to show that the kinetochore protein MAST/Orbit — the single CLASP orthologue in Drosophila is an essential component for microtubule subunit incorporation into fluxing $\mathrm{K}$-fibres.

CLASPs belong to an expanding class of proteins that track along the plus ends of growing microtubules (called +TIPs). They are thought to be involved in regulating microtubule dynamics during both interphase and mitosis ${ }^{3}$. In marked contrast with other +TIPs, CLASPs are unique because they bind to kinetochores throughout mitosis in the absence or presence of attached microtubules 4,5 . We have previously shown that inhibiting CLASP function induces spindles to collapse and causes abnormal chromosome congression, suggesting that CLASP is involved in regulating microtubule dynamics, particularly within K-fibres 5,6 .

To examine this issue in detail, we depleted MAST/Orbit in Drosophila S2 cells stably expressing green fluorescent protein (GFP)-tagged $\alpha$-tubulin (S2T cells ${ }^{7}$ ) by RNA interference (RNAi). This system has several advantages for such studies. First, MAST/Orbit is the only Drosophila orthologue of CLASPs, which eliminates possible redundant functions between CLASP1 and 2. Henceforth, the term 'CLASP' will be used when referring to MAST/Orbit. Second, K-fibres in live S2T cells are robust and can be easily discerned (Fig. 1a). Also, spindle formation has been characterized in detail in these cells ${ }^{8}$. Importantly, in S2T cells the formation of K-fibres is often initiated by kinetochores. As a result, it is not unusual to find $\mathrm{K}$ fibres that formed from the kinetochore without a relationship to an existing centrosome/aster

4Correspondence should be addressed to C.L.R. (e-mail rieder@wadsworth.org).

Note: Supplementary Information is available on the Nature Cell Biology website.

COMPETING FINANCIAL INTERESTS

The authors declare that they have no competing financial interests. 
(see Supplementary Information, Movie 1a) ${ }^{8}$. Together these features provide a unique opportunity for studying how depleting CLASP affects the formation and maintenance of Kfibres during mitosis in animals.

After $72 \mathrm{~h}$ of RNAi treatment, S2T cells are depleted of CLASP (> 95\%; Fig. 1b) ${ }^{6}$. As in controls, in the absence of CLASP centrosomes were often positioned on opposite sides of the nucleus before nuclear envelope breakdown (NEB; Figs 1a, c). After NEB, K-fibres rapidly formed on chromosomes positioned between the separated centrosomes (compare Fig. 1a with c). However, unlike in controls, once formed in CLASP-depleted cells the spindle and its associated K-fibres gradually and progressively shortened (Fig. 1c; also see Supplementary Information, Movie 1b). This confirms earlier conclusions that interfering with CLASP induces bipolar spindles to collapse, providing direct evidence for the shortening of K-fibres during this process. Interestingly, the initial rate of spindle collapse in CLASP-depleted cells was about $1.2 \mu \mathrm{m} \mathrm{min}^{-1}$, or $\sim 0.6 \mu \mathrm{m} \mathrm{min}^{-1}$ for each half spindle ( $n=5$; Fig. 1e and Table 1$)$. However, this rate was not linear with time, implying the presence of a resistant force to spindle shortening. This resistance could arise from varying degrees of CLASP depletion, which could lead to heterogeneity in the severity of the phenotype. However, there is good evidence (for example, see ref. ${ }^{9}$ ) that both microtubule motor molecules and the intrinsic dynamic properties of microtubules are required to maintain spindle bipolarity. Thus, a variable resistance to collapse after CLASP RNAi more likely arises from varying structure of forming spindles combined with different activities of motor proteins such as KLP61F/Eg5, members of the Kinesin- 5 family ${ }^{10}$, which work to maintain spindle bipolarity. This scenario is supported by the observation that spindle collapse in yeast STU1 mutants (the Saccharomyces cerevisiae CLASP) can be rescued by doubling the dosage of Cin8p, the counterpart of KLP61F/Eg5 in this organism ${ }^{11}$.

Our initial observations confirmed that CLASP activity is not required for the genesis of Kfibres. In fact, it was not unusual in CLASP-depleted cells to see K-fibres form, as described above for controls, by a mechanism intrinsic to kinetochores 8 (Fig. 1c). However, as spindles collapsed in CLASP-depleted cells, mature K-fibres not connected to a spindle pole shrank as well (Fig. 1d). This not only reveals that CLASP becomes essential for maintaining K-fibres after they reach a particular maturation stage, but also that K-fibre shortening in cells lacking CLASP occurs by a mechanism intrinsic to the kinetochore or K-fibre and not the spindle poles. In this regard, we recently postulated that CLASPs regulate the dynamics of K-fibres by mediating microtubule subunit incorporation at the kinetochore ${ }^{5}$. If this is true, mature K-fibres in CLASP-depleted cells should not exhibit microtubule subunit flux ${ }^{12}$.

To directly test this prediction, we photobleached short stretches of individual K-fibres in control and CLASP-depleted S2T cells. In control metaphase cells, photobleached marks moved poleward at a rate of $0.70 \pm 0.15 \mu \mathrm{m} \mathrm{min}^{-1}(n=5$; Fig. 2a, e and Table 1). However, in CLASP-depleted cells the bleached mark remained stationary as the spindle gradually collapsed (Fig. 2b, e). CLASPs are components of the kinetochore corona and are the last proteins known to become incorporated into this structure ${ }^{5,13}$. As a result, depleting CLASPs does not affect the targeting of other kinetochore proteins, including CENP-A, CENP-B, CENP-C, CENP-E, Polo, Zw10, CLIP-170 and dynein ${ }^{5,6}$. Thus, it is likely that the absence of microtubule subunit flux in CLASP-depleted cells is due to the direct involvement of CLASP.

To confirm that the effects observed in CLASP-depleted cells are not due to a general perturbation of the kinetochore-microtubule interface, we repeated our photobleaching experiments in cells depleted of either cytoplasmic dynein - another corona protein ${ }^{14}$ - or EB1, which is also a tracking protein delivered to the kinetochore on elongating microtubules ${ }^{15}$. As previously reported, when dynein heavy chain is knocked down, S2T cells 
still form bipolar spindles with properly aligned chromosomes that are delayed in metaphase ${ }^{7,8}$. However, as in plants, the spindle poles in dynein-depleted S2T cells were frequently splayed (Fig. $2 \mathrm{c}$; also see ref. ${ }^{8}$ ). As reported by others ${ }^{16}$, we also found that depleting EB1 in S2T cells led to a severe reduction of astral microtubules and bipolar spindles that were shorter than those of controls (compare Figs 2a and d). Importantly, however, photobleached marks on K-fibres in both dynein- or EB1-depleted cells exhibited persistent poleward motion with rates similar to controls (Fig. 2a-e and Table 1). Together, these results provide compelling evidence that the lack of microtubule subunit flux in CLASP-depleted cells is due specifically to the depletion of CLASP. This, in turn, means that CLASP is required for microtubule subunit addition into mature K-fibres, and in its absence spindles collapse because K-fibres progressively shorten by microtubule subunit loss at their minus ends. Here it is noteworthy that a similar phenotype occurs when vertebrate metaphase cells are treated with low doses of Taxol, which similarly inhibits subunit incorporation into kinetochore microtubule plus ends, but not their removal at the pole ${ }^{17}$. Nevertheless, as CLASP family members are also integral centrosome components 4,5 , it is possible that CLASP depletion also affects microtubule minus-end dynamics.

To examine this possibility, and to also directly determine the role of CLASP at kinetochores, we severed the connection between kinetochores and spindle poles in S2T cells by cutting mature K-fibres with a laser. As shown previously by us 8 (and by others using different systems $18,19,20$ ), when K-fibres in control cells were severed, the fragment that remained connected to the pole (the P-fragment) rapidly disassembled towards the pole at $21.80 \pm 0.35$ $\mu \mathrm{m} \min ^{-1}$. In contrast, the fragment that remained attached to the kinetochore (the K-fragment) re-grew to its original length at $0.78 \pm 0.17 \mu \mathrm{m} \mathrm{min}^{-1}$ (Fig. 3a and Table 1; also see Supplementary Information, Movie $2 \mathrm{a})^{8}$.

As in control cells, when mature K-fibres were severed in CLASP-depleted cells, the Pfragments rapidly disassembled towards the spindle pole (Fig. 3b and Table 1; also see Supplementary Information, Movie 2b). Thus, CLASP depletion does not modify the dynamics of free K-fibre microtubule plus ends created by laser microsurgery. However, in marked contrast to controls, in all 30 CLASP-depleted cells examined, the K-fragments did not change length (that is, re-grow) during the course of the experiment, even as the spindle gradually collapsed (Fig. 3b).

We have previously shown that severed K-fibre re-growth in control S2T cells occurs because the new microtubule minus ends remain stable, whereas microtubule subunits continue to be incorporated into kinetochore microtubule plus ends ${ }^{8}$. Therefore, in control or CLASPdepleted cells the machinery for removing subunits from microtubule minus ends on Kfragments must not be present. As a result, depleting CLASP has no discernible impact on microtubule minus-end dynamics. Accordingly, when we repeated these severing experiments in dynein- or EB1-depleted cells, the results were indistinguishable from the behaviour of Kfragments in control cells (Fig. 3a-e and Table 1, also see Supplementary Information, Movies $2 \mathrm{c}, \mathrm{d})$. Together, these results reveal that K-fragments in CLASP-depleted cells do not elongate, and bleach marks do not move poleward, because CLASP is required for kinetochore-mediated microtubule subunit incorporation into mature (fluxing) K-fibres (Fig. 4). This conclusion is consistent with the observation that CLASP binds to tubulin subunits in vitro (S. Gouveia, $\mathrm{P}$. Sampaio, R. Reis and C. E. Sunkel, unpublished observations).

In Drosophila and most probably other systems ${ }^{21}$, K-fibres that form from the kinetochore do so by a two-step process. First, short microtubule stubs associated with the kinetochore corona begin to elongate by subunit addition at their kinetochore-attached plus ends. This continues until the fibre reaches its mature length, at which time this steady state length is maintained until the onset of anaphase. Both of these processes require subunit addition into microtubule 
plus ends at the kinetochore. However, our data reveal that these two processes occur by different mechanisms. Because K-fibres in CLASP-depleted S2T cells still form by microtubule elongation from the kinetochore, this process is independent of CLASP. In contrast, the mechanism that maintains the length of the K-fibre after it forms, which is based on microtubule subunit flux, clearly involves CLASP. This means that the properties of forming and mature K-fibres differ. In this regard it is possible that CLASP becomes important for kinetochore microtubule dynamics only after the flux machinery becomes engaged. For example, the switch between CLASP-independent K-fibre formation and CLASP-dependent $\mathrm{K}$-fibre maintenance may occur as the molecules responsible for removing subunits accumulate at microtubule minus ends and become operative.

KLP10A, a member of the Kinesin-13 family, has been implicated in K-fibre shortening during mitosis in Drosophila embryos by mediating removal of microtubule subunits at the poles ${ }^{2}$. Its human orthologue, Kif2a, is also required for spindle bipolarity ${ }^{9}$. A molecular view is therefore emerging for how K-fibres elongate and shorten to allow for chromosome motion and position changes in mature spindles: CLASP is involved in regulating microtubule subunit incorporation into K-fibres at the kinetochore, whereas proteins such as KLP10A/Kif2a regulate microtubule depolymerization at the poles (Fig. 4). Any imbalance in the activity of these proteins affects K-fibre length. This leads to changes in the position of the chromosomes relative to the poles and spindle equator, while also establishing spindle length. In crane flies and grasshopper spermatocytes, the shortening of K-fibres occurs exclusively by enhancing the rate of subunit removal near the spindle pole without dampening the rate of incorporation at the kinetochore 22,23 . However, in vertebrates and Drosophila, K-fibre shortening occurs primarily by dampening microtubule subunit incorporation at the kinetochore without changing the rate of subunit removal at the pole $24-26$. Our data suggest that in these latter systems, switches between K-fibre shortening and elongation, required for poleward and awayfrom-the-pole kinetochore motion 27,28 , are regulated by changes in the functional state of CLASPs. Finally, we found that mature K-fibres not connected to a spindle pole shorten in CLASP-depleted cells. This implies that the stable microtubule minus ends of a growing Kfibre in S2T cells switch, at some point in their maturation, into a depolymerization state independent of their association with a centrosome. This most probably occurs as the minus ends recruit molecular factors involved in microtubule subunit removal.

\section{METHODS}

\section{Growing and flattening S2T cells for laser microsurgery and photobleaching}

Drosophila S2T cells were a gift from R. Vale (University of California, San Francisco, CA). The cells were flattened for live cell microscopy analyses, and laser microsurgery/ photobleaching studies were conducted as described 8 . Time-lapse images were collected at $24-26{ }^{\circ} \mathrm{C}$ using either an Olympus IX70 or a Nikon Eclipse TE2000E DIC inverted light microscope equipped with CM350 or CoolSnap HQ cameras, respectively, (Photometrics, Tucson, AZ). Time-lapse data sets were subsequently deblurred using the Softworx 2.5 deconvolution algorithm (Applied Precision, Issaquah, WA). Image sequences were compiled with ImageJ 1.30 (National Institutes of Health) and processed for publication using Adobe Photoshop 6.0.

\section{RNAi}

RNAi depletion of Drosophila CLASP, dynein and EB1 were conducted as described ${ }^{29}$ using published targeting sequences $6,8,16$. Cells were analysed by light microscopy or by immunoblots three days after adding dsRNA to cultures, except for $E B 1$ RNAi, where efficient depletion could only be accomplished after a second treatment with fresh dsRNA for another three days (see Supplementary Information, Fig. S1). 


\section{Supplementary Material}

Refer to Web version on PubMed Central for supplementary material.

\section{Acknowledgements}

We thank G. Goshima, S. Rogers and R. Vale (University of California, San Francisco, CA) for the S2T cells and EB1 antibodies, and C. Sunkel (University of Porto, Portugal) for communicating results before publication. This work was supported by National Institutes of Health grants GMS 40198 (to C.L.R.), GMS 59363 (to A.K.) and a postdoctoral research fellowship from Fundação para a Ciência e a Tecnologia of Portugal (SFRH/BPD/1159/2002 to H.M.).

\section{References}

1. Mitchison TJ. Polewards microtubule flux in the mitotic spindle: evidence from photoactivation of fluorescence. J Cell Biol 1989;109:637-652. [PubMed: 2760109]

2. Rogers GC, et al. Two mitotic kinesins cooperate to drive sister chromatid separation during anaphase. Nature 2004;427:364-370. [PubMed: 14681690]

3. Carvalho P, Tirnauer JS, Pellman D. Surfing on microtubule ends. Trends Cell Biol 2003;13:229-237. [PubMed: 12742166]

4. Lemos CL, et al. Mast, a conserved microtubule-associated protein required for bipolar mitotic spindle organization. EMBO J 2000;19:3668-3682. [PubMed: 10899121]

5. Maiato H, et al. Human CLASP1 is an outer kinetochore component that regulates spindle microtubule dyanmics. Cell 2003;113:891-904. [PubMed: 12837247]

6. Maiato H, et al. MAST/Orbit has a role in microtubule-kinetochore attachment and is essential for chromosome alignment and maintenance of spindle bipolarity. J Cell Biol 2002;157:749-760. [PubMed: 12034769]

7. Goshima G, Vale RD. The roles of microtubule-based motor proteins in mitosis: comprehensive RNAi analysis in the Drosophila S2 cell line. J Cell Biol 2003;162:1003-1016. [PubMed: 12975346]

8. Maiato H, Rieder CL, Khodjakov A. Kinetochore-driven formation of kinetochore fibers contributes to spindle assembly during mitosis in animals. J Cell Biol. 10.1083/jcb.200407090

9. Ganem NJ, Compton DA. The KinI kinesin Kif2a is required for bipolar spindle assembly through a functional relationship with MCAK. J Cell Biol 2004;166:473-478. [PubMed: 15302853]

10. Lawrence CJ, et al. A standardized kinesin nomenclature. J Cell Biol 2004;167:19-22. [PubMed: 15479732]

11. Yin H, You L, Pasqualone D, Kopski KM, Huffaker TC. Stu1p is physically associated with $\beta$-tubulin and is required for structural integrity of the mitotic spindle. Mol Biol Cell 2002;13:1881-1892. [PubMed: 12058056]

12. Sharp DJ. MAST sails through mitosis. Curr Biol 2002;12:R585-R587. [PubMed: 12225678]

13. Desai A, et al. KNL-1 directs assembly of the microtubule-binding interface of the kinetochore in $C$. elegans. Genes Dev 2003;17:2421-2435. [PubMed: 14522947]

14. Hoffman DB, Pearson CG, Yen TJ, Howell BJ, Salmon ED. Microtubule dependent changes in the assembly of microtubule motor proteins and mitotic spindle checkpoint proteins at kinetochores. Mol Biol Cell 2001;12:1995-2009. [PubMed: 11451998]

15. Tirnauer JS, Canman JC, Salmon ED, Mitchison TJ. EB1 targets to kinetochores with attached, polymerizing microtubules. Mol Biol Cell 2002;13:4308-4316. [PubMed: 12475954]

16. Rogers SL, Rogers GC, Sharp DJ, Vale RD. Drosophila EB1 is important for proper assembly, dynamics, and positioning of the mitotic spindle. J Cell Biol 2002;158:873-884. [PubMed: 12213835]

17. Waters JC, Mitchison TJ, Rieder CL, Salmon ED. The kinetochore microtubule minus-end disassembly associated with poleward flux produces a force that can do work. Mol Biol Cell 1996;7:1547-1558. [PubMed: 8898361]

18. Czaban BB, Forer A, Bajer AS. Ultraviolet microbeam irradiation of chromosomal spindle fibres in Haemanthus katherinae endosperm. I. Behaviour of the irradiated region. J Cell Sci 1993;105:571578. [PubMed: 8408286] 
19. Forer A, Spurck T, Pickett-Heaps J. Ultaviolet microbeam irradiations of spindle fibers in crane-fly spermatocytes and newt epithelial cells: resolution of previously conflicting observations. Protoplasma 1997;197:230-240.

20. Gordon, GW. Thesis. Univ. Pennsylvania; 1980. The Control of Mitotic Motility as Influenced by Ultraviolet Microbeam Irradiation of Kinetochore Fibers.

21. Wadsworth P, Khodjakov A. E pluribus unum: towards a universal mechanism for spindle assembly. Trends Cell Biol 2004;14:413-419. [PubMed: 15308207]

22. Chen W, Zhang D. Kinetochore fibre dynamics outside the contex of the spindle during anaphase. Nature Cell Biol 2004;6:227-231. [PubMed: 15039774]

23. LaFountain JR Jr, Cohan CS, Siegel AJ, LaFountain DJ. Direct visualization of microtubule flux during metaphase and anaphase in crane-fly spermatocytes. Mol Biol Cell 2004;15:5724-5732. [PubMed: 15469981]

24. Mitchison TJ, Salmon ED. Poleward kinetochore fiber movement occurs during both metaphase and anaphase-A in newt lung cell mitosis. J Cell Biol 1992;119:569-582. [PubMed: 1400593]

25. Brust-Mascher I, Scholey JM. Microtubule flux and sliding in mitotic spindles of Drosophila embryos. Mol Biol Cell 2002;13:3967-3975. [PubMed: 12429839]

26. Maddox P, Desai A, Oegema K, Mitchison TJ, Salmon ED. Poleward microtubule flux is a major component of spindle dynamics and anaphase A in mitotic Drosophila embryos. Curr Biol 2002;12:1670-1674. [PubMed: 12361570]

27. Skibbens RV, Skeen VP, Salmon ED. Directional instability of kinetochore motility during chromosome congression and segregation in mitotic newt lung cells: a push-pull mechanism. J Cell Biol 1993;122:859-875. [PubMed: 8349735]

28. Khodjakov A, Rieder CL. Kinetochores moving away from their associated pole do not exert a significant pushing force on the chromosome. J Cell Biol 1996;135:315-327. [PubMed: 8896591]

29. Maiato H, Sunkel CE, Earnshaw WC. Dissecting mitosis by RNAi in Drosophila tissue culture cells. Biol Proced 2003;5:153-161. 

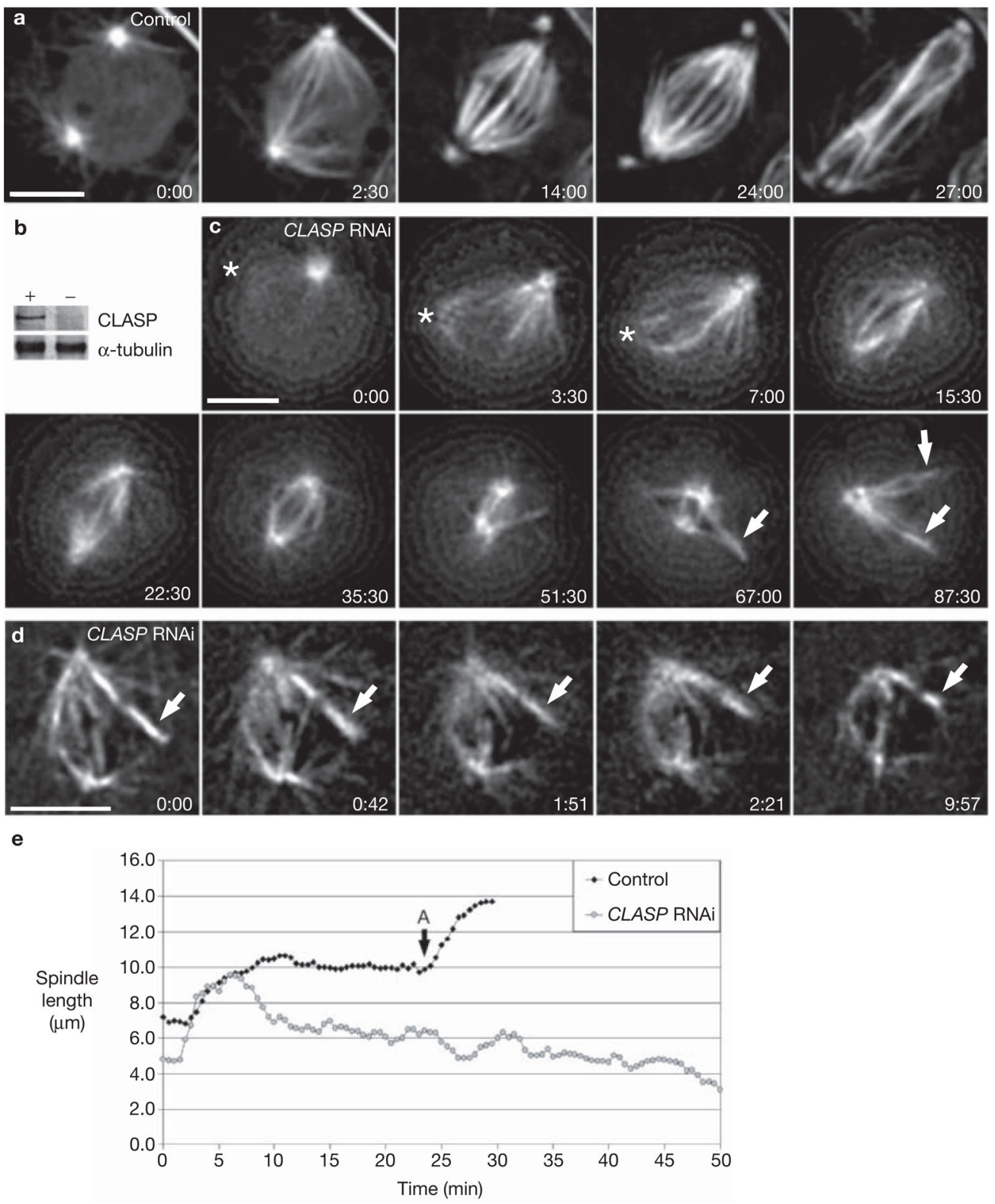

Figure 1.

CLASP is required for maintaining spindle and kinetochore fibre length in Drosophila S2T cells. (a) Mitotic progression in a control cell stably expressing GFP- $\alpha$-tubulin. (b) Western blot showing the depletion of Drosophila CLASP by RNAi (-) compared with control S2T cells (+). Endogenous $\alpha$-tubulin levels are shown as a loading control of protein extracts derived from $10^{6}$ cells per lane. (c) Spindle collapse in a Drosophila S2T cell depleted of CLASP by RNAi. Note that several kinetochore fibres were generated by kinetochores (arrows) as the spindle collapsed. Asterisks indicate a centrosome not visible in some focal planes during the early recording stages. (d) Mature K-fibres, not attached to a centrosome/spindle pole (arrow), also shorten as the spindle collapses in cells depleted of CLASP. Time is shown in min:sec; scale bar represents $5 \mu \mathrm{m}$. (e) Spindle length over time for the control (a) and CLASP-RNAidepleted (c) cells. 'A' indicates the onset of anaphase in the control cell in the chart. Movies of $\mathbf{a}$ and $\mathbf{c}$ can be found in the Supplementary Information. 

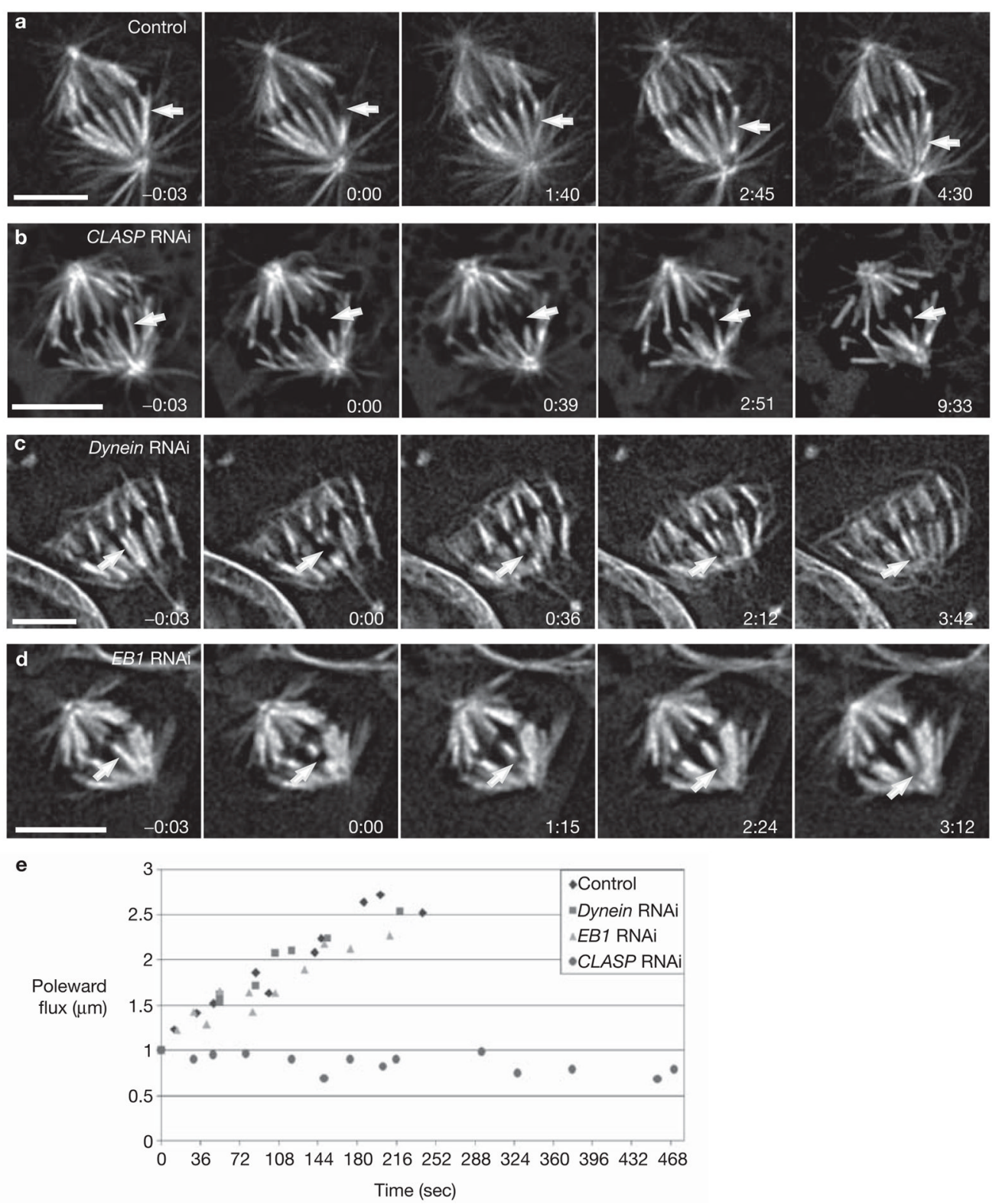

Figure 2.

CLASP is required for microtubule poleward flux. (a) Control cell showing the poleward motion (microtubule subunit flux) of a photobleached region (arrow) on a K-fibre. (b) In cells depleted of CLASP, photobleached marks on K-fibres (arrow) do not move poleward as the spindle gradually collapses. (c, d) Photobleached regions on K-fibres in dynein- or EB1depleted cells, respectively, flux poleward (arrow) at normal rates. Time is shown in min:sec; scale bar represents $5 \mu \mathrm{m}$. (e) Kinetic traces of poleward flux, as assayed from the motion of photobleached regions on K-fibres, from two different cells per condition. 

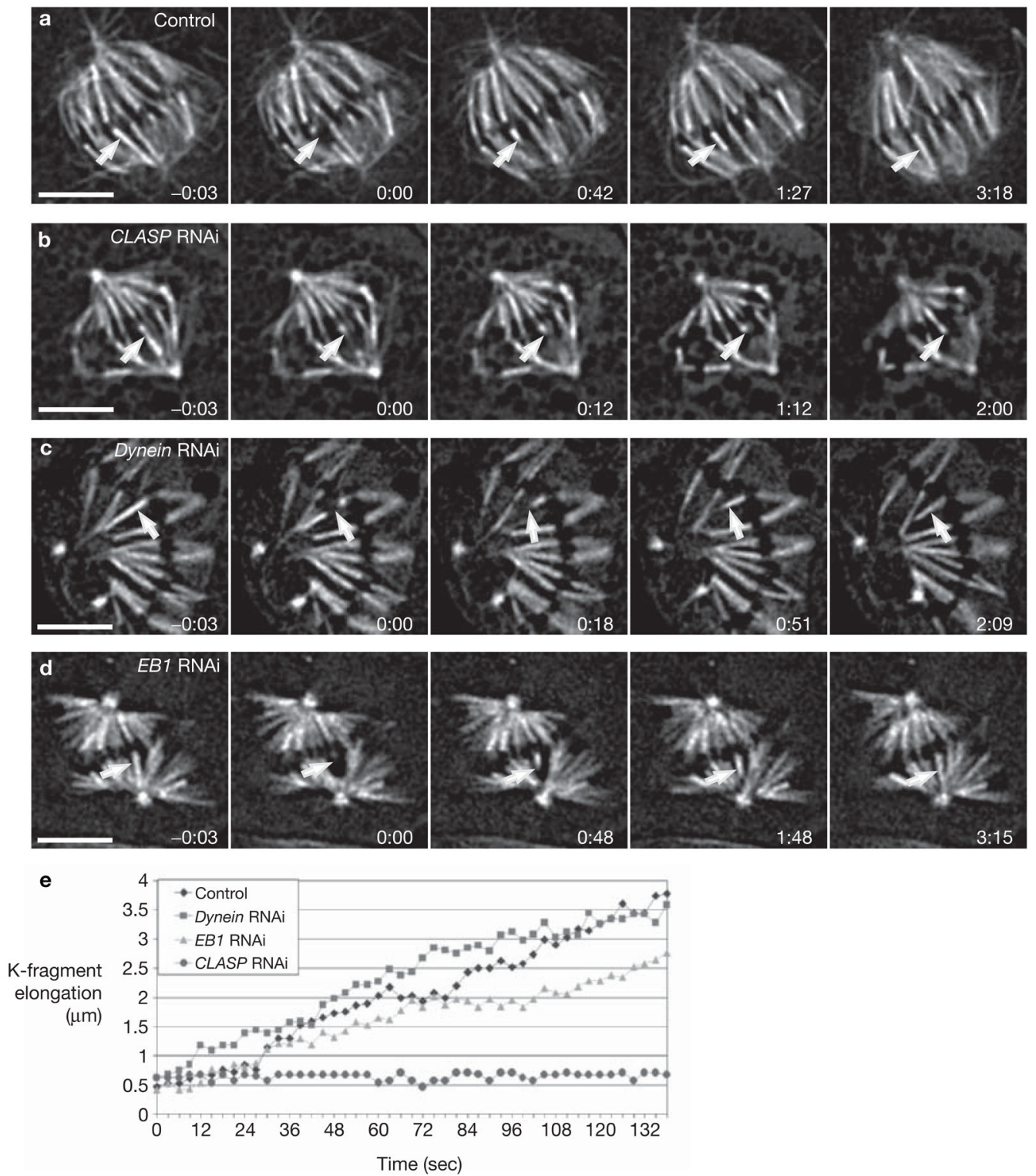

Figure 3.

CLASP is required for subunit incorporation into K-fibre microtubules at their kinetochoreattached plus ends. (a) Control cell showing the re-growth (arrow) of a K-fibre after severing by laser microsurgery. (b) Similar to a but in a cell depleted of CLASP by RNAi. The severed K-fibre (arrow) does not re-grow even as the spindle gradually collapses. (c, d) K-fibres severed in dynein- or EB1-depleted cells, respectively, re-grow (arrow) at the same rates seen in control cells. Time is shown in min:sec; scale bar represents $5 \mu \mathrm{m}$. (e) K-fragment elongation curves after laser microsurgery for the cells shown in the previous panels. Movies of $\mathbf{a}-\mathbf{d}$ can be found in the Supplementary Information. 
a

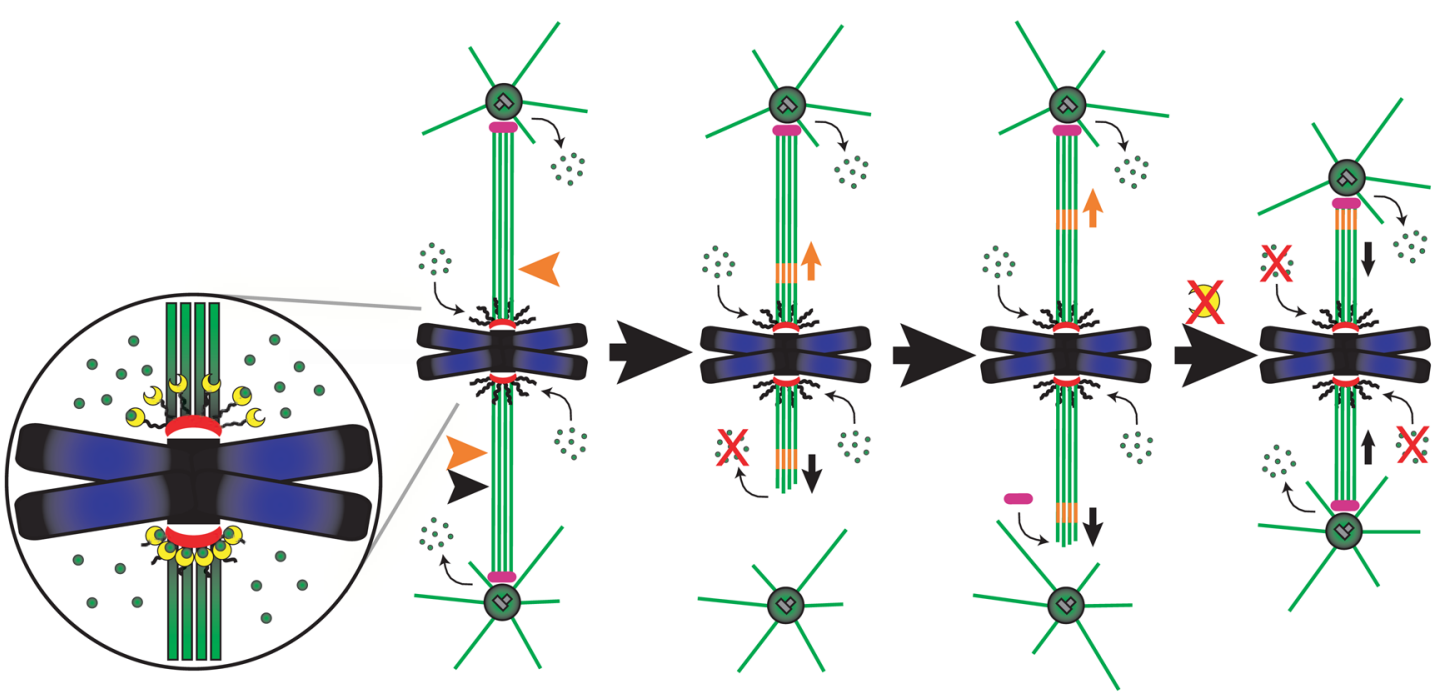

Figure 4.

Schematic depicting the role of CLASP at kinetochores. (a) CLASP (yellow) mediates the incorporation of tubulin subunits (green) into kinetochore microtubule plus-ends at kinetochores. When a mature K-fibre is photobleached (top orange arrow b-d) the subunits move, or 'flux', poleward through the fibre and are removed near the poles. When a K-fibre is photobleached (bottom orange arrow in $\mathbf{b}$ ) and then severed (black arrow) closer to the pole, the fibre re-grows towards the pole (c-d) by microtubule subunit incorporation at the kinetochore but not at the newly created microtubule minus ends, which are stable. Once the re-growing fibre matures, factors are recruited to its microtubule minus ends (d) (most probably Kinesin-13 family members such as KLP10A/Kif2a; pink) that allow for (and maybe power) subunit flux. In the absence of CLASP (e), the incorporation of subunits into mature K-fibre microtubule plus-ends is shut down, which inhibits flux. However, as microtubule minus-end depolymerization still occurs near the poles, the K-fibres shorten and the spindle collapses. 


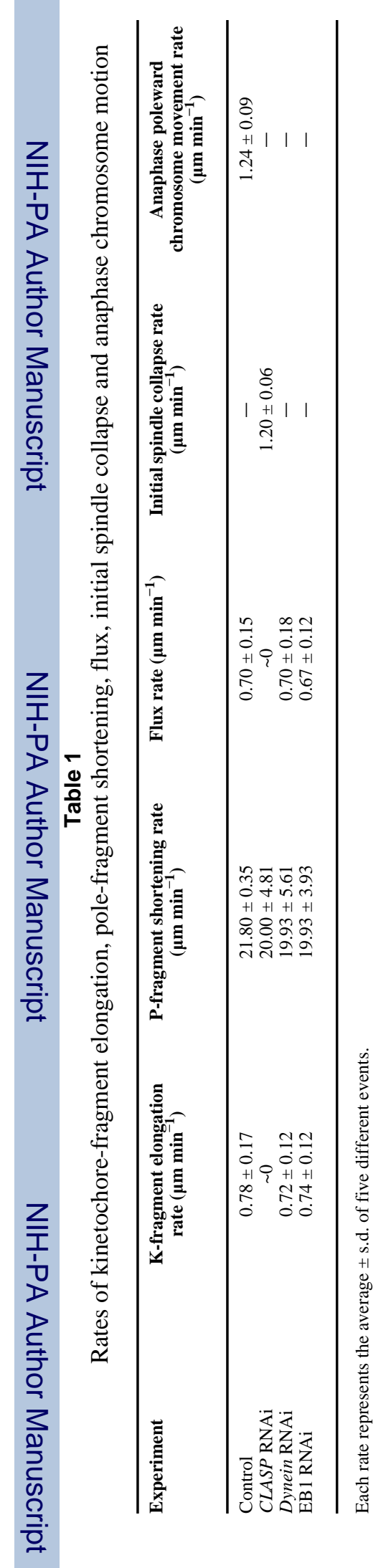

Nat Cell Biol. Author manuscript; available in PMC 2008 December 5. 